\title{
Minisymposium: molecular imaging
}

\author{
Peter Brader
}

Received: 19 July 2010 /Revised: 6 August 2010 / Accepted: 7 August 2010 /Published online: 7 September 2010

(C) Springer-Verlag 2010

Medical imaging has experienced rapid technological development over the last two decades and now plays a central role in clinical radiology. Concomitantly, molecular imaging has emerged combining the disciplines of molecular-cell biology, chemistry and multimodality optical, radiotracer and MR imaging. Molecular imaging is the in vivo characterisation and measurement of biological processes that occur at a cellular and molecular level at a macroscopic level of resolution. This is in contrast to the current conventional, anatomically based radiology [1]. Molecular imaging will play a major role in the near future, not only within the scope of basic research, but also in clinical diagnostics and in therapy-monitoring. It will allow clinicians not only to determine the exact localisation of a disease or lesion, but also to show the expression and activity of specific molecules (proteases, kinases) and biological processes (apoptosis, angiogenesis, and metastasis), the behaviour of diseases and/or response to therapies. This information is expected to have a major impact in early diagnosis, individualized treatment and the development of new therapies, as well as in general on our understanding how diseases arise.

Clinicians rely on radiology to identify abnormal tissue, to assist in planning diagnostic or curative surgical procedures, in staging the extent of disease, to assist in choosing the best systemic therapy as well as for monitoring the effect of therapy and disease progression. Radiology provides detailed information about disease localization, size, metastases and involvement of critical anatomical

P. Brader $(\bowtie)$

Department of Radiology, Division for Molecular and Gender Imaging, Medical University Vienna,

Währinger Gürtel 18-20,

1090 Vienna, Austria

e-mail: peter@brader.md structures. In daily clinical routine these questions are being answered through the application of traditional anatomical imaging methods such as US, CT and MRI.

Although these methods still represent the mainstay of clinical imaging, it has become clear that the acquisition of molecular and physiological information by nuclear magnetic resonance and optical imaging technologies could vastly enhance our ability to fight different diseases.

Emerging genomic and proteomic technologies have the potential to transform the way in which these diseases are managed clinically. Molecular imaging is poised to play a central role in this transformation, because it will allow the integration of molecular and physiological information specific to each patient with anatomical information obtained by conventional imaging methods. The hope is that clinical molecular imaging will one day be used to achieve the following:

(1) The detection of molecular or physiological alterations that signal the presence of certain diseases when they are still at a curable stage. For example, there is an obvious incentive for developing technologies that detect cancer at its earliest stages. In most cases, detection of stage 1 cancers is associated with a $>90 \%$ 5 -year survival rate. When lesions are detected at an even earlier (at the premalignant) stage, treatment is often curative. Conventional anatomical imaging techniques typically detect cancers when they are a centimetre or greater in diameter, at which point they already consist of $>10^{9}$ cells (including circulating and microscopic metastatic deposits). Molecular imaging is expected to play an important role in this setting, because it will allow sensitive and specific monitoring of key molecular targets and host responses associated with early events in carcinogenesis. 
(2) The ability to evaluate and adjust treatment protocols in real time. $\left[{ }^{18} \mathrm{~F}\right]$ FDG-PET imaging has been successful for staging and therapy assessment. Nevertheless, there is a need for continued research to develop better imaging agents that more specifically monitor cell growth and cell death as a means to follow treatment response. As an example, this continuing effort has led to the development of radiolabelled thymidine analogues that may serve as more useful markers of cell proliferation in clinical practice.

(3) The ability to streamline the drug development process. The development of new therapeutics is expensive, time-consuming and often requires vast numbers of patients. These factors all contribute to the final cost of developing and validating new therapies as well as obtaining regulatory approval for clinical use. Molecular imaging has the potential to improve the efficiency and cost-effectiveness of drug development programs. Imaging-based biomarkers (specific molecular targets or biological cancer processes) can be used in all phases of the drug development process. Molecular imaging can help identify new efficacy endpoints that are more easily monitored than currently used endpoints, such as histological analyses of biopsies.

The purpose of this minisymposium is to provide an up-todate overview of this rapidly growing field and to establish a connection to paediatric radiology. The articles of this minisymposium provide important and relevant definitions and background information about the principles of molecular imaging. It describes in detail different imaging technologies, but also first clinical applications are discussed in the context of possible clinical translation with respect to paediatric radiology. Finally, the necessary conditions for radiology are described and discussed to meet the challenge of this young but further expanding field.
The recent and concomitant advances described in this minisymposium will place medical imaging at the centre of medicine. It is therefore important and necessary for radiology to engage, in particular the younger generation of radiologist, to be more involved in this multidisciplinary research field by a number of actions such as those already started by the European Society of Radiology (ESR) [2].

The editor of this minisymposium thanks all the authors for their support and contributions and the effort taken preparing a review article during their busy research and academic life.

The implication for radiology, as it is for almost all of medicine, is to begin thinking at the molecular level. Although most of the imaging techniques discussed in this minisymposium are in preclinical or early clinical phases, paediatric radiology will benefit greatly from these methods. Especially when looking at the leading causes of death in children (Congenital Anomalies and Neoplasia, WHO for Germany 2001) paediatric radiology will benefit in the efforts to detect and diagnose these diseases, to target therapies and to monitor results at the molecular level.

Molecular imaging is the key technology for accomplishing these goals. Imaging practitioners should think of themselves primarily as biologists whose task is to image what is happening functionally and structurally at the molecular level. Although the challenges are significant, the opportunities for molecular imaging are enormous as they will be the future of imaging.

\section{References}

1. Weissleder R, Mahmood U (2001) Molecular imaging. Radiology 219:316-333

2. Grenier N, Sardanelli F, Becker CD et al (2009) Development of molecular imaging in the European radiological community. Eur Radiol 19:533-536 